\title{
A relationship between inhibition of the acoustic startle response and the protection of prepulse processing
}

\author{
CHRISTOPHER M. NORRIS and TERRY D. BLUMENTHAL \\ Wake Forest University, Winston-Salem, North Carolina
}

\begin{abstract}
The relationship between prepulse inhibition of the startle eyeblink reflex and the processing of the prepulse was tested by measuring both the startle eyeblink response and the accuracy of identification of the prepulse in college students. In Experiment $1(N=26)$, startle stimuli were 100-dB(A), 50-msec-duration noise pulses, preceded on some trials by low- or high-frequency 65- $\mathrm{dB}(\mathrm{A})$ tone prepulses at stimulus onset asynchronies (SOAs) of 30-800 msec; in Experiment $2(N=21)$, prepulses were 50 - or $200-\mathrm{Hz}$ vibrotactile pulses to the hand, at SOAs of 50-800 msec. Average prepulse identification accuracy was significantly higher on trials during which startle magnitude was inhibited than on trials during which startle magnitude was not inhibited, at all SOAs. This simultaneous occurrence of prepulse inhibition and higher prepulse identification accuracy shows that the inhibition of the startle response by the prepulse coexists with a decrease in degree to which the startle stimulus (or response) interrupts the preattentive processing of the prepulse.
\end{abstract}

Prepulse inhibition (PPI) of startle is a reliable finding that has received considerable study. Graham $(1979,1992)$ stated that one effect of the startle response is to interrupt ongoing processing, and the function of PPI is to protect the processing of the prepulse from this interruption. That is, prepulse onset initiates two mechanisms: one concerned with processing the prepulse completely enough to identify it, and the other serving to protect this processing from interruption. Previous researchers have shown that manipulations of prepulse intensity, rise time, duration, signal value, interest, and so on, result in variations in PPI. Although their primary motivation was not necessarily an evaluation of Graham's theory, those previous researchers have treated prepulse processing as an independent variable, with startle modification measured at various levels of this variable. However, those previous studies did not directly measure the processing of the prepulse as a dependent variable. The scarcity of reports using a direct test of the protection of preattentive processing, such as that attempted in the present paper, is surprising given the large number of publications in the last two decades in which this protective mechanism is postulated or inferred. The objective of the present research was to test Graham's theory of protection of preattentive processing by assessing both the inhibition of the startle

Portions of this research were presented by the first author to the Department of Psychology at Wake Forest University in partial fulfillment of the requirements for the Masters degree. Portions of this research were presented at the Society for Psychophysiological Research Meetings in Toronto, Canada, October 1995. Correspondence should be addressed to T. D. Blumenthal, Department of Psychology, Wake Forest University, Winston-Salem, NC 27109 (e-mail: blumen@ wfu.edu). response and the processing of the prepulse. The former can tell us that a defensive mechanism has been activated, but the latter is needed to tell us what effect that defensive mechanism has on preattentive processing of the prepulse.

The startle response is a brainstem reflex elicited by the sudden presentation of a sufficiently intense stimulus. It is likely that startle evolved as a defensive response (Davis, 1984), since its behavioral pattern consists of a number of reactions that are usually considered to be protective in nature, such as eyelid closure, limb flexion, and a transient increase in sympathetic output (e.g., increased heart rate). The most common stimuli employed to elicit startle include broadband noise bursts (Blumenthal, 1988), puffs of air (Hackley \& Graham, 1987), mechanical taps to the forehead (Delpezzo \& Hoffman, 1980), electrical stimulation applied to the supraorbital region of the face (Boelhouwer, Teurlings, \& Brunia, 1991), and intense flashes of light (Yates \& Brown, 1981). As a rule, startle can be generated in all mammals and even in some species of insects (Davis, 1984; Riede, 1993; Wyttenbach \& Hoy, 1993).

The presentation of, withdrawal of, or change in another stimulus at a stimulus onset asynchrony (SOA) between 30 and $500 \mathrm{msec}$ prior to the delivery of the startle stimulus may inhibit startle magnitude (Anthony, 1985). This PPI of startle is one of the most robust findings in psychophysiology. The neurological pathways underlying both the elicitation and the modification of the startle reflex have been described in animal models (Davis, Gendelman, Tischler, \& Gendelman, 1982; Swerdlow \& Geyer, 1993). Prepulse modification of startle has been shown to be determined by activity in the pedunculopontine tegmental area (PPTg) of the midbrain (Swerd- 
low \& Geyer, 1993). This area projects to the nucleus reticularis pontis caudalis (nRPC), which is considered to be the area in the pons where the startle response is initiated (Davis, 1984). These studies suggest that activation of the nRPC produces startle responses, and activation of the PPTg inhibits these startle responses. PPI can be potentiated by several stimulus modifications that would be expected to increase neurological activity, such as an increase in prepulse intensity, duration, or number (Anthony, 1985; Blumenthal, 1995; Blumenthal \& Creps, 1994; Blumenthal \& Gescheider, 1987).

The reduction of the acoustic startle response by a prepulse is not due to activation of the middle ear reflex by the prepulse or to peripheral masking of the startle stimulus, since PPI can be shown when the startle-eliciting stimulus is in a modality that is either the same as or different from that of the prepulse (Anthony, 1985; Blumenthal \& Gescheider, 1987; Boelhouwer et al., 1991). The fact that PPI occurs with prepulses that are much lower in intensity than the startle stimulus eliminates the possibility of forward masking of the startle stimulus by the prepulse. PPI is also not due to learning or conditioning, since the effect of the prepulse can be seen on the first prepulse-startle stimulus pairing (Graham, 1975).

Graham $(1975,1979,1992)$ observed that the time window for PPI (30-500 msec) is similar to the time required for stimulus recognition (Massaro, 1970) and suggested that PPI may be a sensorimotor gating phenomenon that prevents the processing of the prepulse from being interrupted. Graham's theory can be summarized as follows: When a prepulse is presented, the subject begins to automatically process the prepulse in an attempt to "find out what it is." This processing stage has been referred to as preattention since it occurs after stimulus detection but before stimulus recognition. This processing stage, while crucial to stimulus recognition, is also vulnerable to interruption by extraneous stimuli and/or motoric responses. To prevent the interruption of preattention, a "preattentive defense mechanism" acts to suppress responding to extraneous stimuli during this processing stage. Therefore, if a startle stimulus is presented while the subject is engaged in processing the prepulse, responding to that startle stimulus will be inhibited by the preattentive defense mechanism, thus decreasing the interruption of prepulse processing that would otherwise be caused by the startle stimulus. If the startle stimulus is presented after prepulse recognition has occurred (after about $500 \mathrm{msec}$ ), the preattentive defense mechanism is no longer needed and either does not become active or "shuts off." Therefore, responding to the startle stimulus is no longer inhibited, and the result is a response that very much resembles a startle reflex elicited by a startle stimulus presented alone (i.e., without a prepulse).

A great deal of research has been published in which the properties of the prepulse have been varied and the effects of these variations on startle responding have been assessed. This can be interpreted as manipulation of the prepulse as an independent variable, with the subsequent measurement of PPI as a dependent variable.
These data can be used to evaluate Graham's theory, on the basis of the assumption that the manipulation of prepulse properties results in more or different processing for some prepulses relative to other prepulses. However, a different test of Graham's hypothesis could involve the measurement of not only PPI as a dependent variable but of prepulse processing as well. When the mechanism that protects prepulse processing has been activated (as shown by PPI), the processing of the prepulse should be improved relative to when startle is not inhibited.

The purpose of the present research was to evaluate Graham's theory of PPI by measuring not only the extent to which a prepulse inhibits the startle response but also the extent to which the processing of the prepulse is protected from interruption. The latter was investigated by having the subject decide if a prepulse had been presented and, if it had, whether it was a high-frequency or a lowfrequency prepulse. On the basis of Graham's $(1975,1979$, 1992) hypothesis of protection of preattentive processing, accuracy of prepulse identification should be lower on trials during which the tone prepulse is followed by a startle stimulus than on trials during which the tone is presented alone, due to the interruption of prepulse processing caused by the startle system. If PPI is an indication that the protective mechanism has been activated, then accuracy of prepulse identification should be higher on trials during which the startle response is inhibited than on trials during which the startle response is not inhibited.

\section{EXPERIMENT 1}

\section{Method}

Subjects. The subjects were 26 college students ( 18 females, 8 males) at Wake Forest University who participated for extra credit in their introductory psychology course. Average age was 18 years 10 months (range $=18$ years 4 months to 20 years 0 months). All subjects reported having no significant hearing loss in their medical history. The subjects were divided into two groups of 13 subjects each, as described below.

Stimuli and Apparatus. The startle-eliciting stimulus was a 50 -msec-duration, $100-\mathrm{dB}(\mathrm{A})$ burst of broadband noise $(20 \mathrm{~Hz}$ to $20 \mathrm{kHz}$ ) with a rise/fall time of less than $1 \mathrm{msec}$. The two prepulses employed were 20 -msec-duration, $65-\mathrm{dB}(\mathrm{A})$ tones with a rise/fall time of $5 \mathrm{msec}$ and frequencies of 300 or $800 \mathrm{~Hz}$ in Group $1(n=13)$ and 800 or $1300 \mathrm{~Hz}$ in Group $2(n=13)$. The use of two groups with similar but not identical tasks was based on an effort to avoid an effect of absolute prepulse frequencies. That is, both groups were presented with prepulses that differed in frequency, but the "high-frequency" prepulse for one group was the same as the "low-frequency" prepulse for the other group.

On prepulse trials, the onset of the prepulse preceded startle stimulus onset by either $30,120,480$, or $800 \mathrm{msec}$. Four control trials were also used: two where the startle stimulus occurred alone (no prepulse) and two where either the low-frequency or the high-frequency tone occurred alone (no startle stimulus). The experimental session consisted of five blocks of 12 trials per block ( 60 trials) in which each of the 12 stimulus conditions was presented once per block, in random order within each block. Stimulus presentation was controlled by a Macintosh SE computer and a MacPacq MP10 interface.

The startle stimulus was produced by a Coulbourn S81-02 noise generator, and the prepulse tones were produced by two separate Coulbourn S81-06 precision signal generators. Each of the three 
stimuli was then gated through separate Coulbourn S84-04 selectable envelope-shaped rise/fall gates, amplified by a Coulbourn S82-24 audio-mixer amplifier, and delivered to the subjects over Telephonics TDH-49P stereo headphones.

The intensity of each stimulus was calibrated by a Quest Electronics 215 sound level meter and headphone coupler. The corresponding voltages of the stimuli were recorded and checked prior to each experimental session with a Fluke 8050A digital multimeter.

Response measurement and apparatus. SensorMedics miniature $\mathrm{Ag} / \mathrm{AgCl}$ electrodes were used to measure electromyographic (EMG) activity from the periorbital region of the subject's left orbicularis oculi muscle (just below the left eye). EMG activity was amplified by a Coulbourn S75-01 high-gain bioamplifier with filters passing 90 to $250 \mathrm{~Hz}$ and smoothed by a Coulbourn S76-01 contour following integrator with a time constant of $10 \mathrm{msec}$ (Blumenthal, 1994). The gain of the integrated EMG signal was then adjusted by a Coulbourn S79-02 adjustable gain amplifier and sampled by the MacPacq interface for $1 \mathrm{sec}$ at a rate of $1000 \mathrm{~Hz}$ and converted into digital units for storage on the Macintosh SE computer.

A button box $(8 \times 15 \times 5 \mathrm{~cm})$ was used to obtain prepulse identification information. On the top of the button box were three buttons aligned in a row, approximately $3 \mathrm{~cm}$ apart. Each button was labeled "low tone," "high tone," or "no tone." The position of the labels was counterbalanced across subjects. The pulse generated by a buttonpress activated an LED on a Coulbourn S22-04 optically isolated switch input, and the experimenter recorded which button the subject had pressed.

Procedure. Each subject filled out an informed consent form and a health questionnaire. Next, the skin just beneath the subject's left eye was cleaned with an alcohol-saturated cotton swab. A ground electrode was placed on the inner left forearm, and two recording electrodes were placed below the subject's left eye, situated horizontally to one another, with one electrode under the center of the eye and the center of the other electrode approximately $15 \mathrm{~mm}$ temporal to the center of the first electrode. All electrodes were filled with Signa electrode cream.

Following electrode placement, the subject was seated in a sound-attenuated room (background noise less than $30 \mathrm{~dB}[\mathrm{~A}]$ ) and told that he/she would be hearing two tones with different frequencies over the headphones during the experiment. It was explained that these two tones might or might not be paired with a loud "burst of static" and that the amount of time between the tone and the static burst could differ from trial to trial. The subjects were then instructed to press, as quickly but as accurately as possible after the tone was presented, the button on the button box that corresponded to the tone that they thought had been presented on that particular trial ("low tone" or "high tone"). The subjects were instructed to press the "no tone" button if they thought that the static burst had been presented alone. Headphones were then placed on the subject, and the button box was placed in his/her lap. Next, examples of the low-frequency and high-frequency tones (without the startle stimuli) were presented to the subject, to demonstrate the frequency difference between these two tones and to ascertain that the subject could discriminate one tone from the other. None of the subjects had any difficulty making this frequency discrimination. The experimenter then answered any questions that the subject asked, closed the door to the sound-attenuated room, and initiated the experiment. There were 60 trials in the session, with an intertrial interval randomly varying between 15 and $25 \mathrm{sec}$ (20 sec average).

Dependent variables. Startle eyeblink EMG was used to determine startle magnitude and onset latency. The time window in which a startle eyeblink was measured began $20 \mathrm{msec}$ after startle stimulus onset and ended $100 \mathrm{msec}$ after startle stimulus onset. Startle magnitude was obtained by subtracting the EMG level at startle response onset from the peak startle EMG level. If no re- sponse was measured but a response could have been measured on a trial, a magnitude of zero was assigned, so that an acceptable magnitude value was available on each trial. Startle response onset latency was defined as the time between startle stimulus onset and startle eyeblink onset. Latency was averaged across trial blocks within each stimulus condition, but only from trials during which a response was actually measured. That is, if there was no response on a trial, the latency on that trial was treated as missing. Two startle-stimulus-alone control trials were presented in each trial block, and the control value for startle magnitude and latency for a given trial block was quantified as the average from the two control trials in that block. Prepulse identification accuracy was determined for each trial by the response of the subject, either correct or incorrect. In each trial block, each tone was presented alone, without a startle stimulus, to allow for a control measure of prepulse identification in the absence of an interrupting startle stimulus. On a very small number of trials (less than $1 \%$ ), movement artifact prevented the measurement of an eyeblink response. Startle magnitude, startle latency, and prepulse identification accuracy were treated as missing for these trials.

Startle data were analyzed with repeated measures ANOVAs (BMDP4V), with Greenhouse-Geisser corrected degrees of freedom used to determine significance, but standard degrees of freedom are reported, along with epsilon values wherever appropriate. Habituation trends across trial blocks and orthogonal trends across levels of SOA were assessed for startle magnitude by using orthogonal ANOVAs (BMDP2V). Prepulse identification accuracy was assessed with $t$ tests, using a modified Scheffé method to protect against excessive Type I error. Pearson product--moment correlation coefficients were calculated (BMDP3D) between startle responding and prepulse identification accuracy and between the amount of PPI and the amount of interruption of prepulse processing (difference between accuracy on a prepulse trial and that on a tone-alone trial).

The relationship between PPI and preattentive processing was assessed by comparing prepulse identification accuracy on trials during which the startle response was inhibited with accuracy on trials during which the startle response was not inhibited. First, accuracy on each trial was scored as either 1 (correct) or 0 (incorrect). Second, a trial was labeled an inhibition trial (PPI) if startle magnitude was smaller on that trial than on the average of the two control trials in the same trial block. Prepulse trials on which startle responding was not smaller than that on the average of the two control trials in that block were labeled noninhibition trials (no PPI). Third, each of the 10 trials ( 2 trials in each of five blocks) presented at each level of prepulse SOA was placed in a 2 (inhibition) $\times 4$ (SOA) matrix, with each cell containing a minimum of 0 and a maximum of 10 trials from each subject. For a particular subject, the inhibition and the noninhibition data came from different trials, since a particular trial for a given subject could not be in both the inhibition half of the data matrix and the noninhibition half of the matrix. Some subjects contributed more data to the inhibition half of this matrix than to the noninhibition half of this matrix, and this contribution varied from one SOA condition to another. PPI on at least 1 of the 10 trials possible at SOAs of $30,120,480$, and $800 \mathrm{msec}$ was found in $22,25,26$, and 25 of the 26 subjects, respectively. No PPI was found on at least 1 of the 10 trials possible at SOAs of $30,120,480$, and $800 \mathrm{msec}$ in $15,6,11$, and 21 of the 26 subjects, respectively. Fourth, the average prepulse identification accuracy in each cell was calculated. Fifth, the average accuracy was then compared for inhibition trials and noninhibition trials at a single SOA, using $t$ tests. A $t$ test is more conservative than an ANOVA when there is a wide discrepancy in the number of subjects contributing data to the various cells of the data matrix. A modified Scheffé method was used to be still more conservative, given that the inhibition and noninhibition cells were being compared at each SOA (four $t$ tests in all). A significant $t$ test would show that the accuracy of identifying the 
tone was higher on trials during which PPI was found than on noninhibition trials. Finally, prepulse identification accuracy was compared between each prepulse condition and the tone-alone control condition, to evaluate the degree to which the startle stimulus interrupted processing of the prepulse. Again, $t$ tests and a modified Scheffé method were used.

\section{Results}

Startle response magnitude. The two subject groups did not differ significantly in terms of response magnitude on control trials $(F=1.22)$ or in the amount of PPI $(F=1.70)$. Prepulse SOA had a significant effect on startle magnitude $[F(3,72)=17.76, p<.001$, epsilon $=$ .90 ; see Figure 1]. Startle magnitude was inhibited by prepulses at SOAs of 30,120 , and $480 \mathrm{msec}\left[F_{\mathbf{S}}(1,24)=\right.$ $11.02,50.35$, and 26.40 , respectively, $p s<.01$ ], but prepulses at an 800-msec SOA had no significant effect on startle magnitude.

Prepulse identification accuracy. The two subject groups did not differ significantly in terms of prepulse identification accuracy on tone-alone control trials $(F=$ $3.09)$ or in the influence of SOA and PPI on accuracy $(F=$ 1.21). Prepulse identification accuracy was significantly higher on inhibition trials than on noninhibition trials at all SOAs $[t(35)=18.05, t(29)=24.59, t(35)=38.40$, and $t(44)=38.92$, for SOAs of $30,120,480$, and $800 \mathrm{msec}$, respectively, $p \mathrm{~s}<.01$; see Figure 2]. Prepulse identification accuracy was lower in the prepulse condition than in the control condition at SOAs of 30,120 , and $480 \mathrm{msec}$ on inhibition trials and at all SOAs on noninhibition trials $(p s<.01)$. However, this difference from control was always significantly greater on noninhibition trials than on inhibition trials $(p<.01)$.

Correlations between startle responding and prepulse identification accuracy were not significant in any condition, nor were correlations between the amount of PPI and the difference in accuracy between prepulse trials and tone-alone trials.

Habituation of startle and PPI. Startle response magnitude habituated across the session $[F(4,96)=10.30$,

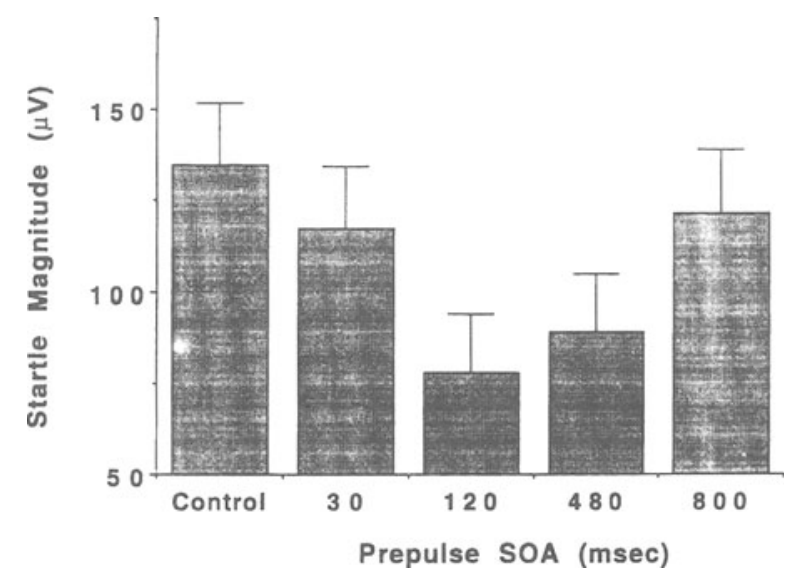

Figure 1. Startle response magnitude in control and prepulse conditions in Experiment 1. Bars represent $1 S E M$.

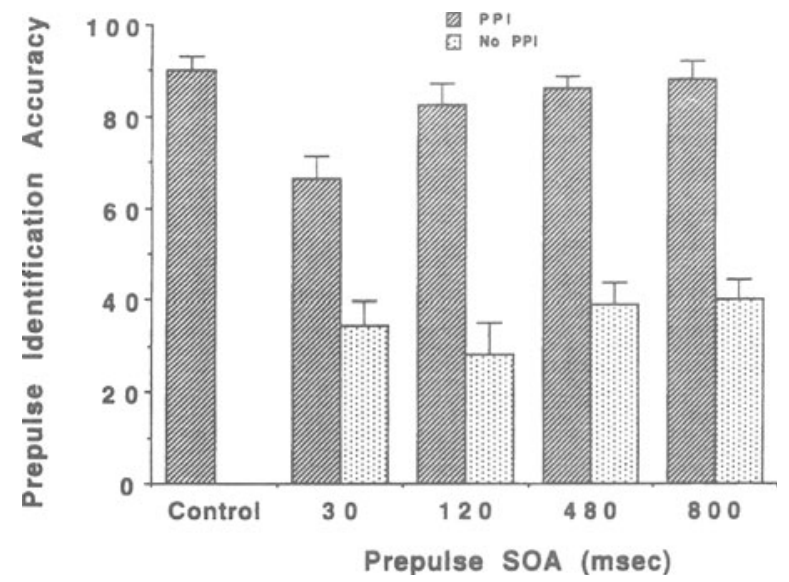

Figure 2. Prepulse identification accuracy in percent for trials during which startle magnitude was either inhibited (PPI) or not inhibited (No PPI), at each prepulse SOA in Experiment 1. Bars represent $1 S E M$.

$p<.001]$, with the linear trend reaching significance $[F(1,24)=21.97, p<.001]$. The trial block variable did not interact with group. The degree to which prepulses inhibited the magnitude of the startle response decreased across trial blocks $[F(4,96)=3.67, p<.01]$, and this decrease was characterized by a linear trend $[F(1,24)=$ $13.80, p<.01]$. The trial block variable did not interact with either SOA or group in this analysis. ${ }^{1}$

Startle response latency. No significant differences were found between the two subject groups in terms of response latency. The effect of a prepulse on startle response latency was quantified as the difference between responding in a prepulse condition and responding in the control condition. Prepulse SOA had a significant effect on startle latency $[F(3,72)=10.32, p<.001]$. Prepulses facilitated startle latency at a 30-msec SOA $[F(1,24)=$ $36.89, p<.001]$ but had no effect on startle latency at other SOAs.

\section{Discussion}

Stimulus effects on startle responding in Experiment 1 were consistent with those found in previous studies. Prepulses inhibited startle magnitude at all SOAs except the longest $(800 \mathrm{msec}$ ), as expected (Anthony, 1985; Graham, 1975). At the shortest SOA of $30 \mathrm{msec}$, the prepulses inhibited startle magnitude and facilitated startle latency, demonstrating the partial independence of these two measures (Graham \& Murray, 1977). Startle response magnitude in the control condition habituated across the session, as expected (Schicatano \& Blumenthal, 1994). PPI decreased as the session progressed, suggesting either that PPI habituates or that PPI is reduced as the response to the startle stimulus habituates (Graham \& Murray, 1977; Graham, Putnam, \& Leavitt, 1975; Lipp, Arnold, Siddle, \& Dawson, 1994).

The processing of the prepulse was partially protected from the interruptive influence of the startle system on those trials on which startle was inhibited, as shown by 
higher prepulse identification accuracy on inhibition trials than on noninhibition trials. These data support Graham's statement that the function of PPI is to protect the perceptual analysis of the prepulse from interruption.

Prepulse identification accuracy was considerably lower in the 30-msec SOA condition than in the tonealone condition, and this may be evidence for the interruption of preattentive processing by the startle system (Graham, 1979). However, an alternative explanation must be considered-that of backward masking. Massaro (1970) has shown that the accuracy of identifying a target tone decreases if the target is followed by a masking tone at an interval of $250 \mathrm{msec}$ or less. Therefore, the startle stimulus used in Experiment 1 may have masked the prepulse, displacing the prepulse from the preperceptual store and interfering with the accurate identification of the prepulse (Massaro, 1970). The possibility that the location of this masking is central and not in the sensory periphery is supported by the finding that the same prepulses that inhibited startle magnitude at short SOAs facilitated startle latency. It is unlikely that both results could occur if the peripheral gain of the stimulus input were attenuated by a masker. Also, backward masking in the auditory periphery has been shown to last for a very short time, less than $15 \mathrm{msec}$ (Pickles, 1982).

The centrally occurring backward-masking explanation is based on the fact that both stimuli used here were acoustic, and this masking would not be present if the prepulse and startle stimulus were in different modalities (Massaro \& Kahn, 1973). To deal with this backwardmasking alternative hypothesis, a second experiment was conducted in which acoustic startle stimuli were preceded by vibrotactile prepulse stimuli presented to the hand. These vibrotactile stimuli have been demonstrated to cause startle inhibition at appropriate SOAs (Blumenthal \& Gescheider, 1987; Blumenthal \& Tolomeo, 1989). When vibrotactile prepulses are presented at a $50-\mathrm{msec}$ SOA before an acoustic startle stimulus, the result is startle facilitation rather than startle inhibition (Blumenthal \& Gescheider, 1987, Blumenthal \& Tolomeo, 1989). This may be due to the different time of arrival of these stimulus-generated neural events at the facial nucleus (Boelhouwer et al., 1991). Therefore, a 50-msec SOA was included in this second experiment, to look at the relationship between prepulse identification accuracy and both startle inhibition and facilitation. Vibrotactile prepulses offer a crossmodal way to study PPI in which prepulse parameters, such as duration, intensity, rise time, and frequency, can be precisely controlled. Also, the use of vibrotactile prepulses is preferred to the use of electrical stimuli, since vibrotactile prepulses involve transduction by sensory receptors (Bolanowski, Gescheider, Verrillo, \& Checkosky, 1988), whereas electrical stimulation may bypass the transduction stage and stimulate neurons directly. This vibrotactile method provides a way to evaluate the effects of prepulses and startle stimuli on prepulse identification accuracy without the possibility of backward masking within a sensory channel.
Specifically, if the decrease in prepulse identification accuracy found at the 30-msec SOA in Experiment 1 is still present at any SOA in Experiment 2, then this decrease cannot be due solely to backward masking.

The use of vibrotactile prepulses can also be used to avoid loudness assimilation, another potential explanation for the effects of startle stimuli on prepulse processing. Perlstein, Fiorito, Simons, and Graham (1993) presented $75-\mathrm{dB}$ prepulse tones paired with either 75- or $110-\mathrm{dB}$ tones at SOAs of 120 or $500 \mathrm{msec}$. In one condition, the task of the subject was to estimate the magnitude of the first tone in a pair. Subjects judged the first tone in a 75- to $110-\mathrm{dB}$ pair to be more intense than when that $75-\mathrm{dB}$ tone was presented alone. This occurred at a $500-\mathrm{msec}$ SOA, but not at a $120-\mathrm{msec}$ SOA. Perlstein et al. (1993) explain this as loudness assimilation, in which the "loudness of pair members shifted toward one another" (p. 356). Filion and Ciranni (1994) found a similar loudness assimilation effect on magnitude estimation of the prepulse stimulus, using $75-$ and $110-\mathrm{dB}$ tones at SOAs of 120 and $500 \mathrm{msec}$. In both of these studies, the processing of the prepulse was facilitated by the presence of the startle stimulus, which would not be expected if the startle system interrupts prepulse processing. Filion and Ciranni (1994) found a significant correlation between the amount of PPI and loudness assimilation at a $120-\mathrm{msec}$ SOA, but not at a $500-\mathrm{msec}$ SOA. They present this as support for Graham's (1992) theory of protection of prepulse processing. However, their data also suggest that the magnitude estimation task could not assess the extent to which the startle system interrupted prepulse processing, since loudness assimilation is in a direction opposite to the effect expected of interruption of prepulse processing. Therefore, investigating the relationship between loudness assimilation and PPI may not be a useful test of Graham's protection hypothesis.

By using prepulses and startle stimuli in different modalities, the relationship between PPI and prepulse identification accuracy could be investigated without the possibility of loudness assimilation or backward masking. Therefore, a second experiment was conducted, in which vibrotactile prepulses were followed on some trials by acoustic startle stimuli at a range of SOAs.

\section{EXPERIMENT 2}

\section{Method}

Subjects. The subjects were 21 students ( 11 females, 10 males) at Wake Forest University who participated for extra credit in their introductory psychology course. Average age was 21 years 10 months (range $=18$ years 6 months to 31 years 4 months). Only subjects who scored as strongly right-handed on a modified version of the Edinburgh Handedness Inventory (Oldfield, 1971) were included in this experiment. No subjects reported problems of hearing loss prior to the experimental session. None of the subjects in Experiment 2 had participated in Experiment 1.

Stimuli, Apparatus, Procedure, and Data analysis. The stimuli, apparatus, procedure, and data analyses were similar to those used in Experiment 1, with several exceptions. Again, the startle-eliciting stimulus was a 50-msec-duration, 100-dB(A) 
burst of broadband noise with a rise/fall time of less than $1 \mathrm{msec}$ (background noise in the testing chamber was less than $30 \mathrm{~dB}[\mathrm{~A}]$ ). The prepulses were $50-$ or $200-\mathrm{Hz}$ vibrations presented to the hand, with a duration of $40 \mathrm{msec}$ and a rise/fall time of $5 \mathrm{msec}$. Each prepulse was presented at SOAs of $50,100,200,400$, or $800 \mathrm{msec}$. Prepulses were generated by two Coulbourn S81-06 precision signal generators gated through separate Coulbourn S84-04 selectable envelope-shaped rise/fall gates and amplified by a Coulbourn S82-24 audio-mixer amplifier. The output of this amplifier activated a Ling 203 Shaker (vibrator) with an attached $3.0-\mathrm{cm}^{2}$ contoured circular contactor, which protruded through a small hole in the desk at which the subject sat. The subject was asked to rest the left hand on the desk through which the vibrator contactor protruded. The positioning of the arm was such that the contoured disc contacted the thenar eminence (the fleshy part of the palm just below the thumb) of the left hand. The subject was asked to relax the hand and not to move it until the end of the session. The vibrator made a slight noise, but this was not detectable when the subject wore the headphones, and the vibrator was covered with the hand.

The task given the subject was to press a button with the right hand corresponding to the vibration presented ("low vibration," "high vibration," or "no vibration"), analogous to the task used in Experiment 1. The session consisted of five blocks of 14 trials per block ( 70 trials), in which each of the 14 stimulus conditions (each prepulse frequency at each SOA paired with startle, each prepulse frequency presented alone, and 2 trials of the startle stimulus presented alone) was presented once per block, in random order within each block.

The relationship between PPI and preattentive processing was assessed in a way analogous to that used in Experiment 1. PPI on at least 1 of the 10 trials possible at SOAs of 50,100,200,400, and $800 \mathrm{msec}$ was found in $15,21,21,21$, and 20 of the 21 subjects, respectively. No PPI was found on at least 1 of the 10 trials possible at SOAs of $50,100,200,400$, and $800 \mathrm{msec}$ in $20,11,3,4$, and 9 of the 21 subjects, respectively.

\section{Results}

Startle response magnitude. Prepulse SOA had a significant effect on the modification of startle magnitude $[F(4,80)=26.24, p<.001$, epsilon $=.45]$. Startle magnitude was inhibited by prepulses at SOAs of 100,200 , 400 , and $800 \mathrm{msec}\left[F_{\mathrm{S}}(1,20)=17.15,23.46,22.88\right.$, and 16.82, respectively, $p s<.001$; see Figure 3]. Prepulses at a 50-msec SOA had no significant effect on startle magnitude $(p<.10)$.

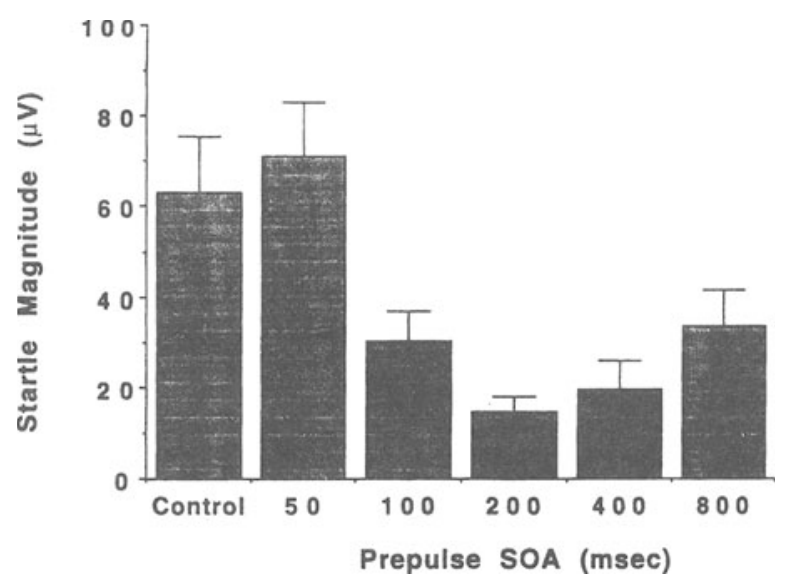

Figure 3. Startle response magnitude in control and prepulse conditions in Experiment 2. Bars represent 1 SEM.

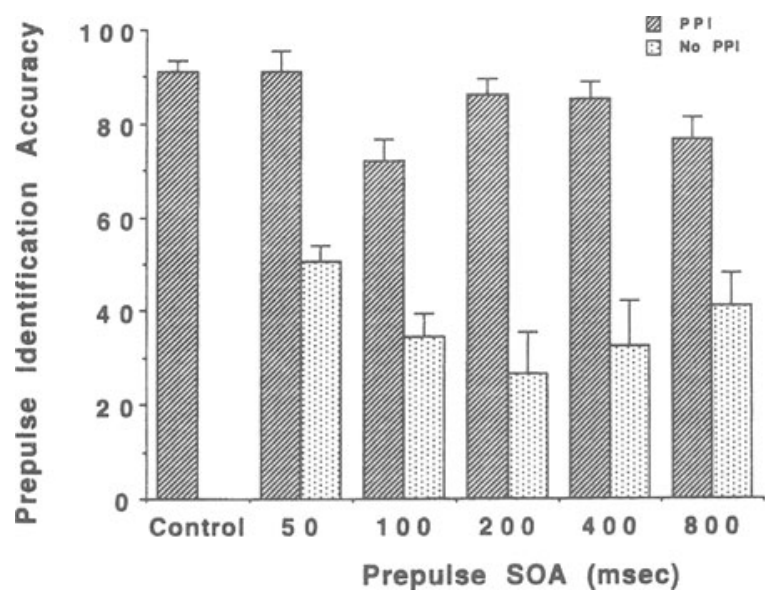

Figure 4. Prepulse identification accuracy in percent for trials during which startle magnitude was either inhibited (PPI) or not inhibited (No PPI), at each prepulse SOA in Experiment 2. Bars represent 1 SEM.

Prepulse identification accuracy. Prepulse identification accuracy was significantly higher on inhibition trials than on noninhibition trials at all SOAs $[t(33)=$ $32.21, t(30)=20.61, t(22)=24.16, t(23)=19.77$, and $t(27)=16.09$, for SOAs of 50,100,200, 400, and $800 \mathrm{msec}$, respectively, $p s<.01$ ] (see Figure 4). Prepulse identification accuracy was lower in the prepulse condition than in the control condition at all SOAs except $50 \mathrm{msec}$ on inhibition trials and at all SOAs on noninhibition trials $(p s<.01)$. However, this difference from control was always significantly greater on noninhibition trials than on inhibition trials $(p<.01){ }^{2}$

Correlations between startle responding and prepulse identification accuracy were not significant in any condition, nor were correlations between the amount of PPI and the difference in accuracy between prepulse trials and tone-alone trials.

Habituation of startle and PPI. Startle response magnitude habituated across the session $[F(4,80)=$ $12.23, p<.001]$, with the linear, quadratic, and cubic trends reaching significance $[F(1,20)=20.20,5.09$, and 7.33 , respectively, $p s<.05]$. The degree to which prepulses inhibited the magnitude of the startle response decreased across trial blocks $[F(4,80)=5.13, p<.01]$, with the linear trend reaching significance $[F(1,20)=$ $11.09, p<.01]$. The trial block effect interacted with prepulse SOA $[F(16,320)=7.44, p<.001]$, in that the inhibition caused by the prepulses at SOAs of $100-800 \mathrm{msec}$ decreased across trial blocks, but the effect of the 50msec SOA prepulse did not change across trial blocks. In fact, when the data were analyzed without the $50-\mathrm{msec}$ SOA conditions, the trial block $\times$ SOA interaction was no longer significant. Also, when the 50-msec SOA data were analyzed alone, no trial block effect was found.

Startle response latency. Startle latency was facilitated by prepulses at an SOA of $50 \mathrm{msec}[F(1,20)=$ $97.27, p<.001]$, with no other prepulses having a significant effect on response latency. 


\section{Discussion}

As in Experiment 1, both startle responding and the amount of PPI decreased across trial blocks. The vibrotactile prepulses were effective inhibitors of acoustic startle at SOAs of $100 \mathrm{msec}$ or more, similar to previous findings (Blumenthal \& Gescheider, 1987; Blumenthal \& Tolomeo, 1989). At all SOAs, average prepulse identification accuracy was lower on trials during which startle was not inhibited than on trials during which startle was inhibited, similar to the results of Experiment 1.

Comparing prepulse identification accuracy in Experiment 2 to that in Experiment 1, accuracy was consistently lower than that in the tone-alone condition at an SOA of $30 \mathrm{msec}$ in Experiment 1 and an SOA of $100 \mathrm{msec}$ in Experiment 2. When vibrotactile prepulses are used, the shortest SOA (50 msec in this experiment) often results in startle facilitation rather than startle inhibition (Blumenthal \& Gescheider, 1987; Blumenthal \& Tolomeo, 1989). This may be due to the different time of arrival of these stimulus-generated neural events at the facial nucleus (Boelhouwer et al., 1991). However, these data suggest that the decrease in accuracy at the 30-msec SOA in Experiment 1 may have been due to a combination of backward masking (Massaro, 1970) and interruption by the startle system. In Experiment 2, a similar decrease in accuracy was seen at a $100-\mathrm{msec}$ SOA, and this could not have been due to backward masking, since the prepulse and startle stimuli were in different sensory modalities.

As was the case in Experiment 1, correlations between measures of startle responding and prepulse identification accuracy were generally not significant. When we look at PPI, we are interested in the difference in responding to the startle stimulus on prepulse trials relative to control trials. This means that increased PPI is represented by an increased difference between these two types of trials. In similar fashion, when we look at the protection of prepulse processing, we are less interested in prepulse identification accuracy on prepulse-startle trials than in the difference in accuracy on prepulse trials relative to tone-alone trials. As accuracy on prepulse trials increases, this difference decreases, indicating less interruption of prepulse processing caused by the startle stimulus. Therefore, we predict that, as PPI increases, the difference in prepulse identification accuracy should approach zero, since increased PPI may be associated with decreased interruption of prepulse processing. As PPI decreases, approaching zero, the difference in prepulse identification accuracy should become increasingly large and negative, reflecting more interruption of prepulse processing caused by the noninhibited startle stimulus or response. Correlational analyses are not useful when one variable stays near zero as the other variable increases or decreases. Since the task used in the present experiments was relatively easy, prepulse identification accuracy was generally very high, and presentation of startle stimuli decreased this accuracy at some SOAs. However, the accuracy data were negatively skewed, not normally distributed, and this deviation from the normal distribution means that correlation should not be used to evaluate these data. That is why we have separated our data into categories, according to the presence or absence of PPI.

\section{GENERAL DISCUSSION}

The novel contribution of the present report is found in the relationship between PPI and prepulse identification accuracy relative to control. In both experiments, this accuracy was higher (closer to control levels) on trials during which the startle response was inhibited than on trials during which the startle response was not inhibited. This suggests that both prepulse processing and PPI may be initiated by the presentation of the prepulse, even if the two processes are dependent upon different underlying mechanisms.

The backward-masking alternative hypothesis discussed above (Massaro, 1970) may have contributed to the reduction of prepulse identification accuracy at a 30msec SOA in Experiment 1. The window of backward masking found by Massaro (1970) was approximately $250 \mathrm{msec}$, whereas the window was narrower in our Experiment 1 . We may have shown a narrower window of backward masking because our masking stimulus, the startle stimulus, was shorter in duration than the stimulus used by Massaro. Backward masking may be one way in which the startle system interrupts the processing of the prepulse, when the prepulse and startle-eliciting stimuli are presented in the same modality. Backward masking cannot explain the lowered prepulse identification accuracy at the 100-msec SOA in Experiment 2, so the interruptive properties of the startle system are not entirely based on backward masking. It may be the case that the startle stimulus interrupted the processing of the prepulses in both experiments, but this interruption was added to by backward masking at the $30-\mathrm{msec}$ SOA in Experiment 1.

These data show that prepulse effects on startle responding are probabilistic, not certain. That is, at a given SOA and in a given trial block, startle magnitude will be more or less likely to be lower on prepulse trials than on control trials. The likelihood of this PPI is greater at some SOAs than at others (e.g., more likely at $120-\mathrm{msec}$ SOA than at $800-\mathrm{msec} \mathrm{SOA}$ ), and it is this overall likelihood that provides the basis for saying that, over the entire session and subject group, prepulses at one SOA result in PPI, whereas prepulses at another SOA do not. However, when the data are inspected at the level of the trial block, we see that a prepulse condition in which PPI is found over the entire study may show some trials on which startle was not inhibited, even at the optimal SOA for PPI. Conversely, a prepulse that does not, overall, inhibit startle might do so on some trial blocks in some subjects. The probability that PPI will be found can be thought of as varying within some distribution, the average value of which is most often reported, and this average is used to say whether or not PPI occurs at a particular SOA. The present data show that the trials that are not in the majority can still be informative. Since the re- 
lationship between PPI and prepulse identification accuracy may depend upon a minority of trials at some SOAs, this accuracy must be studied at the level of the trial block, not over the entire session.

These data support Graham's (1979) hypothesis that the startle system interrupts prepulse processing and that PPI is paired with the protection of the preattentive processing of the prepulse. The reduction in prepulse identification accuracy at a 30-msec SOA in Experiment 1 and a 100-msec SOA in Experiment 2 supports the interruptive properties of the startle system. The difference in prepulse identification accuracy on inhibited and noninhibited trials supports the presence of a defensive mechanism that inhibits startle and also allows the preattentive processing of the prepulse to continue. These data, using prepulse processing as a dependent variable, converge with the findings from studies in which prepulse processing has been manipulated as an independent variable (Anthony, 1985; Blumenthal \& Creps, 1994; Filion, Dawson, \& Schell, 1993; Hackley \& Graham, 1987). In both situations, PPI is associated with more effective prepulse processing. Although the direction of causality cannot be assessed in the present study, these results clearly demonstrate a relationship between PPI and prepulse identification accuracy. Specifically, when the defensive mechanism is activated, as demonstrated by PPI, the prepulse tends to be identified more accurately. This finding is consistent with the possibility that prepulse inhibition of startle plays a role in protecting the preattentive processing of the prepulse.

\section{REFERENCES}

ANTHONY, B. J. (1985). In the blink of an eye: Implications of reflex modification for information processing. In P. K. Ackles, J. R. Jennings, \& M. G. H. Coles (Eds.), Advances in psychophysiology (Vol. 1, pp. 167-218). Greenwich, CT: JAI.

BLUMENTHAL, T. D. (1988). The startle response to acoustic stimuli near startle threshold: Effects of stimulus rise and fall time, duration, and intensity. Psychophysiology, 25, 607-611

BLUmenthal, T. D. (1994). Signal attenuation as a function of integrator time constant and signal duration. Psychophysiology, 31, 201-203.

Blumenthal, T. D. (1995). Prepulse inhibition of the startle eyeblink as an indicator of temporal summation. Perception \& Psychophysics, $57,487-494$

Blumenthal, T. D., \& Creps, C. L. (1994). Normal startle responding in psychosis-prone college students. Personality \& Individual Differences, 17, 345-355.

Blumenthal, T. D., \& Gescheider, G. A. (1987). Modification of the acoustic startle reflex by a tactile prepulse: The effects of stimulus onset asynchrony and prepulse intensity. Psychophysiology, 24, 320-327.

Blumenthal, T. D., \& Tolomeo, E. A. (1989). Bidirectional influences of vibrotactile stimuli on modification of the human acoustic startle reflex. Psychobiology, 17, 315-322.

Boelhouwer, A. J. W., Teurlings, R. F. M. A., \& Brunia, C. H. M. (1991). The effect of an acoustic warning stimulus upon the electrically elicited blink reflex in humans. Psychophysiology, 28, 133 139.

Bolanowski, S. J., Jr., Gescheider, G. A., Verrillo, R. T., \& CheCKOSKY, C. M. (1988). Four channels mediate the mechanical aspects of touch. Journal of the Acoustical Society of America, 84, 1680-1694. DAvIs, M. (1984). The mammalian startle response. In R. C. Eaton
(Ed.), Neural mechanisms of startle behavior (pp. 287-342). New York: Plenum.

Davis, M., Gendelman, D., Tischler, M., \& Gendelman, P. (1982). A primary acoustic startle circuit: Lesion and stimulation studies. Journal of Neuroscience, 2, 791-805.

Delpezzo, E. M., \& Hofrman, H. S. (1980, November 7). Attentional factors in the inhibition of a reflex by a visual stimulus. Science, 210, 673-674.

Filion, D. L., \& Ciranni, M. (1994). The functional significance of prepulse inhibition: A test of the protection of processing theory [Abstract]. Psychophysiology, 31, S46.

Filion, D. L., DAwson, M. E., \& SCHELl, A. M. (1993). Modification of the acoustic startle-reflex eyeblink: A tool for investigating early and late attentional processes. Biological Psychology, 35, 185-200.

GraHAM, F. K. (1975). The more or less startling effects of weak prestimulation. Psychophysiology, 12, 238-248.

GraHAM, F. K. (1979). Distinguishing among orienting, defense, and startle reflexes. In H. D. Kimmel, E. H. van Olst, \& J. F. Orlebeke (Eds.), The orienting reflex in humans (pp. 137-167). Hillsdale, NJ: Erlbaum

Graham, F. K. (1992). Attention: The heartbeat, the blink, and the brain. In B. A. Campbell, H. Hayne, \& R. Richardson (Eds.), Attention and information processing in infants and adults (pp. 3-29). Hillsdale, NJ: Erlbaum.

Graham, F. K., \& MurRay, G. M. (1977). Discordant effects of weak prestimulation on magnitude and latency of the reflex blink. Physiological Psychology, 5, 108-114.

Graham, F. K., Putnam, L. E., \& LeavitT, L. A. (1975). Lead stimulation effects on human cardiac orienting and blink reflexes. Journal of Experimental Psychology: Human Perception \& Performance, 1, 161-169.

HaCKLEY, S. A., \& GRAHAM, F. K. (1987). Effects of attending selectively to the spatial position of reflex-eliciting and reflex-modulating stimuli. Journal of Experimental Psychology: Human Perception \& Performance, 13, 411-424.

Lipp, O. V., ArNold, S. L., Siddle, D. A. T., \& Dawson, M. E. (1994). The effect of repeated prepulse-blink reflex trials on blink reflex modulation at short lead intervals. Biological Psychology, 38, 19-36.

Massaro, D. W. (1970). Perceptual auditory images. Journal of Experimental Psychology, 85, 411-417.

Massaro, D. W., \& KaHN, B. J. (1973). Effects of central processing on auditory recognition. Journal of Experimental Psychology, 97, $51-58$

OLDFIELD, R. C. (1971). The assessment and analysis of handedness: The Edinburgh inventory. Neuropsychologia, 9, 97-113.

Perlstein, W. M., Fiorito, E., Simons, R. F., \& Graham, F. K. (1993). Lead stimulation effects on reflex blink, exogenous brain potentials, and loudness judgments. Psychophysiology, 30, 347-358.

PICKLES, J. O. (1982). An introduction to the physiology of hearing. London: Academic Press.

RIEDE, K. (1993). Prepulse inhibition of the startle reaction in the locust locusta-migratoria (Insecta, Orthoptera, Acridoidea). Journal of Comparative Physiology: Sensory, Neural, \& Behavioral Physiology, 172, 351-358.

Schicatano, E. J., \& Blumenthal, T. D. (1994). Caffeine delays habituation of the human acoustic startle response. Psychobiology, 22, 117-122.

Swerdlow, N. R., \& Geyer, M. A. (1993). Prepulse inhibition of acoustic startle in rats after lesions of the pedunculopontine tegmental nucleus. Behavioral Neuroscience, 107, 104-117.

Wyttenbach, R. A., \& Hoy, R. R. (1993). Prepulse effects on the acoustic startle response of field crickets. Society for Neuroscience Abstracts, 19, 70.9.

YATES, S. K., \& BROWN, W. F. (1981). Light-stimulus evoked blink reflex: Methods, normal values, relation to other blink reflexes, and observations in multiple sclerosis. Neurology, 31, 272-281.

\section{NOTES}

1. The trial block factor was not significant for prepulse identification accuracy in Experiments 1 and 2. This means that accuracy did 
not decrease across trial blocks. In fact, there was a slight but nonsignificant increase in accuracy as the session progressed. This could be due to a practice effect; however, it might also support the relationship between PPI and accuracy, since, as PPI decreased across the session, accuracy increased slightly.

2. Another type of analysis was conducted on the data from both of these experiments, looking at the amount of PPI on correct versus incorrect trials. This analysis was analogous to the one conducted on accuracy as a function of PPI, except that these two variables were "reversed." That is, trials in Experiment 1 were divided into a $2 \times 4$ matrix, with correct trials or incorrect trials at each of the four SOAs. In Experiment 2, a $2 \times 5$ matrix was used, with five SOAs. On each trial, the amount of PPI was calculated as the difference between startle responding on that prepulse trial and the average response magnitude on the two control trials in that trial block for that subject. The average amount of PPI at each SOA was then calculated from the available subject data at each SOA. The mean PPI for correct and incorrect trials at each SOA were compared with $t$ tests, using a modified Scheffé procedure to be conservative. The number of subjects contributing to the correct half of these matrices ranged from 17 to 26 out of 26 in Experiment 1 and from 20 to 21 out of 21 in Experiment 2. The number of subjects contributing to the incorrect half of these matrices ranged from 3 to 10 out of 26 in Experiment 1 and from 1 to 4 out of 21 in Experiment 2. Given this low number of data points in some cells, these data must be interpreted with caution, and they are presented in this note for the interest of the reader. Given that caveat, in Experiment 1, PPI was significantly more pronounced on trials on which the prepulse was correctly identified than on trials on which prepulse identification was incorrect, at SOAs of 30 and $480 \mathrm{msec}$, but not at 120- and 800-msec SOAs. In Experiment 2, PPI was more pronounced on correct than incorrect trials at SOAs of 200 and $400 \mathrm{msec}$, but not at $100-$ or $800-\mathrm{msec}$ SOAs. At an SOA of $50 \mathrm{msec}$, prepulses facilitated startle significantly more on incorrect than on correct trials

(Manuscript received September 21, 1995; revision accepted for publication February 19, 1996.) 